\title{
Constrained Filtering with Contact Detection Data for the Localization and Registration of Continuum Robots in Flexible Environments
}

\author{
Stephen Tully, Andrea Bajo, George Kantor, Howie Choset, Nabil Simaan
}

\begin{abstract}
This paper presents a novel filtering technique that uses contact detection data and environmental stiffness estimates to register and localize a robot with respect to an a priori 3D surface model. The algorithm leverages geometric constraints within a Kalman filter framework and relies on two distinct update procedures: 1) an equality constrained step for when the robot is forcefully contacting the environment, and 2) an inequality constrained step for when the robot lies in the freespace of the environment. This filtering procedure registers the robot by incrementally eliminating probabilistically infeasible state space regions until a high likelihood solution emerges. In addition to registration and localization, the algorithm can estimate the deformation of the surface model and can detect false positives with respect to contact estimation. This method is experimentally evaluated with an experiment involving a continuum robot interacting with a bench-top flexible structure. The presented algorithm produces an experimental error in registration (with respect to the end-effector position) of 1.1 $\mathrm{mm}$, which is less than 0.8 percent of the robot length.
\end{abstract}

\section{INTRODUCTION}

Minimally invasive surgery (MIS) reduces patient recovery time and post-surgical infection. Robotic MIS has the potential to improve surgical accuracy and enable deeper anatomical reach through small incisions [1]-[3], but at a price of limited sensory feedback and visualization of the surgical field. In most surgical disciplines, physicians typically rely on expertise to interpret preoperative images and to correlate them with the surgical reality. More recently, live medical imaging has been used to guide surgeons to targeted anatomical locations (e.g. fluoroscopy [4], MRI [5], CT [6], and 3D ultrasound [7]). Unfortunately, many of these imaging modalities either have a limited field of view, are incompatible with robotic systems or MIS, and/or emit prolonged radiation.

A recent alternative to using medical images for surgical guidance is to perform model-based image-guided surgery. The goal is to create a virtualized rendered view of the operation for visual feedback by combining tool tracking with a 3D model of the anatomical structure [8]. Commercial examples of this type of sensor-guided feedback are Ensite NavX (St Jude Medical, St Paul, MN, USA) and Carto XP/CartoMerge (Bio-Sense Webster, Diamond Bar, CA,

S. Tully is with the Electrical and Computer Engineering Department at Carnegie Mellon University, Pittsburgh, PA 15213, USA. G. Kantor and $\mathrm{H}$. Choset are with the Robotics Institute at Carnegie Mellon University, Pittsburgh, PA 15213, USA, stully@ece, kantor@ri, choset@cs\}. cmu.edu. S. Tully is supported by Army Research Office Grant \#W911NF1010343.

A. Bajo and N. Simaan are with the Mechanical Engineering Department at Vanderbilt University, Nashville, TN 37240, USA, \{andrea.bajo, nabil.simaan\}@vanderbilt.edu. A. Bajo and N. Simaan are supported by NSF Career \#IIS-1063750.

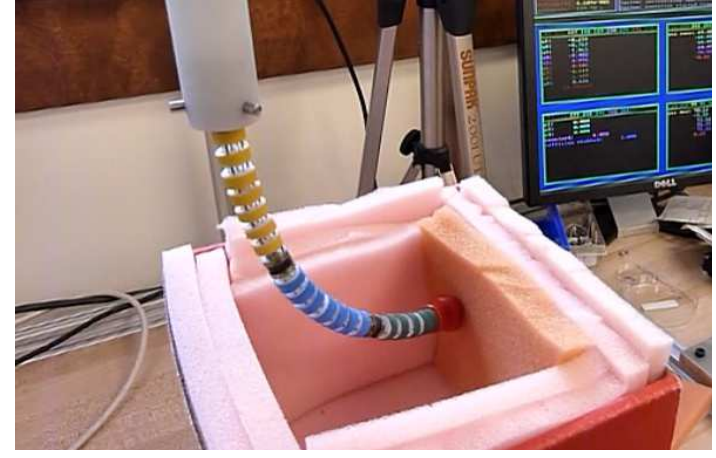

Fig. 1. The experimental setup used for evaluating our constrained filtering algorithm in the presence of surface deformation.

USA), which are used for cardiac mapping and ablation. For successful model-based image-guidance, the tracked surgical tool must be accurately registered to the coordinate frame of the a priori 3D surface model [9]. A common approach is to use an iterative closest point (ICP) algorithm [10] that minimizes the difference between two point clouds. Unfortunately, this can be computationally intensive in the case of aligning a large number of points [11]. Another method uses ultrasound to register a surgical tool inside of a heart model using a particle filter [12]. In general, achieving sufficiently accurate registration is a challenging task, especially in the presence of tissue deformation.

This paper introduces a novel equality constrained filtering approach that enables the automatic registration and localization of a compliant surgical robot operating in a deformable environment. The central idea of our method is that when environmental contact is detected between the robot and the surface [13], this information can be used to construct an equality constraint on the state space of a Kalman filter. Applying this constraint to the filter will eliminate infeasible regions of the state space, and when a sufficient number of constraints are applied, the true state will emerge that defines the registration of the surgical tool to the deformable surface.

The advantage of this probabilistic approach is its potential accuracy. By formulating this problem as a filtering task, we are in a sense using a nonlinear state estimator as a means to fuse all available sources of information (robot compliance models, material stiffness, kinematic models, tracker measurements, and prior surface models) with the hope of resolving the most likely solution. Another advantage is that the estimation algorithm naturally provides error-bounds, thus one can easily determine if the registration result can be trusted. Lastly, there are two favorable by-products of 
our algorithm: the estimation of surface deformation and the detection of false-positives with respect to contact estimation.

The ideas presented in this paper build upon our previous work that investigated the use of constrained filtering for surgical registration [14]. Our previous work ignored any possible contact between the robot and its environment, though, and it was also assumed that the surrounding surface was rigid. Our new approach significantly improves upon our previous work by relaxing these assumptions.

The main contribution of this work is the novel application of equality constrained Kalman filtering to the problem of surgical registration within a flexible environment. Our approach uses elastic force models within a Kalman filter to define constraints on the system and uses an iterative pseudomeasurement update method to apply each constraint during the registration process. We have validated our approach experimentally with a bench-top experiment using a continuum robot designed for MIS [3], [15], see Fig. 1.

\section{Nonlinear Constrained Kalman Filtering}

Constrained Kalman filtering is the problem of correcting or constraining the Kalman update or Kalman prediction to account for known constraints on the state vector [16]. With constrained filtering, the uncertainty of the state estimate can be significantly reduced. This is particularly useful for our surgical application because preoperative surface models can directly provide geometric constraints. In this section, we will discuss our algorithms to handle equality and inequality constraints within a Kalman filter. Then, in the following section, we will discuss the novel application of these algorithms to surgical estimation.

\section{A. Equality Constrained Kalman Filtering}

When an equality constraint, such as,

$$
g\left(\mathbf{x}_{k}\right)=c
$$

can be defined with respect to the $N \times 1$ system state $\mathbf{x}_{k}$, equality constrained Kalman filtering [17]-[21] seeks to update the Kalman filter mean and covariance $\left(\hat{\mathbf{x}}_{k \mid k}, \mathbf{P}_{k \mid k}\right)$ to incorporate this information, thus producing a new estimate $\left(\hat{\mathbf{x}}_{k \mid k}^{+}, \mathbf{P}_{k \mid k}^{+}\right)$.

In [22], Simon and Chia present a method for equality constrained Kalman filtering in which linear constraints are incorporated into the Kalman filter update by projecting the unconstrained estimate onto the constraint hyperplane. Another method, which in [20], [21] is shown to be equivalent to the projection approach, is to use a pseudo-measurement technique that simulates a perfect measurement to enforce equality constraints [17]-[19].

We will now introduce our iterative algorithm, which is related to the pseudo-measurement approaches developed in [17]-[19]. Our approach to this problem is similar to the iterative method discussed by Julier in [23] and is in some ways similar to Wang's maximum likelihood approach, presented in [19], due to its use of iteration.

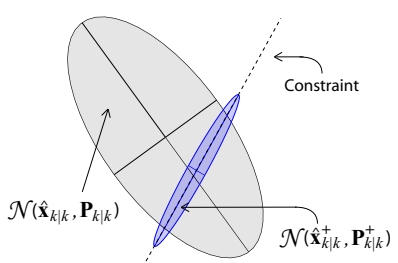

a)

a)

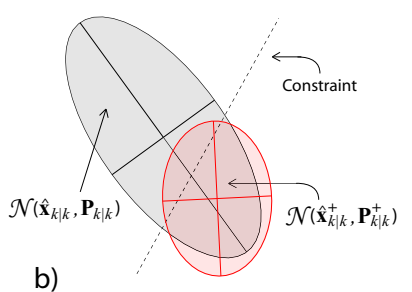

Fig. 2. With equality constrained Kalman filtering, seen in (a), the probability density function (PDF) will lie on the constraint. With inequality constrained filtering, seen in (b), the updated PDF will encompass the feasible region.

At a given time-step $k$, we seek to update the mean of the filter with the vector $\mathbf{x}$ that minimizes the following cost function,

$$
\begin{aligned}
\hat{\mathbf{x}}_{k \mid k}^{+}= & \underset{\mathbf{x}}{\operatorname{argmin}}\left[\hat{\mathbf{x}}_{k \mid k}-\mathbf{x}\right]^{T} \mathbf{P}_{k \mid k}^{-1}\left[\hat{\mathbf{x}}_{k \mid k}-\mathbf{x}\right] \\
& \text { subject to } g(\mathbf{x})=c .
\end{aligned}
$$

The purpose of this optimization problem is to replace the Kalman estimate with the state $\mathbf{x}$ that, out of the set of states that comply with the equality constraint, has the highest likelihood.

An equivalent problem to Eq. 2 can be defined instead using unconstrained optimization, as follows,

$\hat{\mathbf{x}}_{k \mid k}^{+}=\underset{\mathbf{x}}{\operatorname{argmin}}\left[\hat{\mathbf{x}}_{k \mid k}-\mathbf{x}\right]^{T} \mathbf{P}_{k \mid k}^{-1}\left[\hat{\mathbf{x}}_{k \mid k}-\mathbf{x}\right]+\sigma_{g}^{2-1}\|g(\mathbf{x})-c\|$.

where $\sigma_{g}^{2}$ is defined to be the measurement noise of the simulated pseudo-measurement. This optimization problem is solved for $\sigma_{g}^{2} \rightarrow 0^{+}$to simulate a perfect measurement in order to strictly enforce the equality constraint. GaussNewton's method can be used to minimize this cost function numerically. The recursive procedure to compute the state vector follows,

$$
\begin{aligned}
\mathbf{x}_{0} & =\hat{\mathbf{x}}_{k \mid k} \\
\mathbf{K}_{i} & =\mathbf{P}_{k \mid k} \mathbf{G}_{i}^{T}\left[\mathbf{G}_{i} \mathbf{P}_{k \mid k} \mathbf{G}_{i}^{T}\right]^{-1} \\
\mathbf{x}_{i+1} & =\hat{\mathbf{x}}_{k \mid k}+\mathbf{K}_{i}\left[c-g\left(\mathbf{x}_{i}\right)-\mathbf{G}_{i}\left(\hat{\mathbf{x}}_{k \mid k}-\mathbf{x}_{i}\right)\right],
\end{aligned}
$$

where $\mathbf{G}$ is the Jacobian of $g(\mathbf{x})$ linearized about $\mathbf{x}_{i}$. Finally, we update the mean of the Kalman filter $\hat{\mathbf{x}}_{k \mid k}^{+}$with the state $\mathbf{x}_{i}$ at convergence. The reason that our method relates to the pseudo-measurement technique for constrained filtering which was presented in [17]-[19] is that we are essentially performing an iterated extended Kalman filter (IEKF) update step [24] with a zero variance pseudo-measurement $g(\mathbf{x})$.

After updating the mean of the filter, the covariance matrix can then be updated with the conventional Kalman covariance update equation,

$$
\begin{aligned}
\mathbf{K} & =\mathbf{P}_{k \mid k} \mathbf{G}^{T}\left[\mathbf{G P}_{k \mid k} \mathbf{G}^{T}+\epsilon\right]^{-1} \\
\mathbf{P}_{k \mid k}^{+} & =\mathbf{P}_{k \mid k}-\mathbf{K} \mathbf{G} \mathbf{P}_{k \mid k},
\end{aligned}
$$

where $\mathbf{G}$ is the Jacobian of $g(\mathbf{x})$ linearized about the updated state mean $\hat{\mathbf{x}}_{k \mid k}^{+}$. Additionally, as shown in Eq. 4, we adjust the Kalman gain by inflating the simulated measurement noise by $\epsilon$. This can help prevent numerical issues that would occur with a singular covariance matrix, as discussed in [23]. 
The benefit of our approach is that, despite a nonlinear constraint, the updated state estimate is forced to exactly comply with the constraint equation in Eq. 1. This is due to the convergence of the optimization problem that we defined in Eq. 3. This compares to the use of an extended Kalman filter (EKF) for the pseudo-meaurement update, which would not necessarily place the new estimate on the constraint due to linearization issues.

The example in Fig. 2-(a) illustrates our equality constrained filtering method for a two-dimensional problem. In Fig. 2-(a), a constraint tells us that the true state must lie on the hyperplane shown with a dotted line. The algorithm updates the Gaussian probability density function (PDF) associated with the Kalman estimate to lie along that dimension according to the constraint.

\section{B. Inequality Constrained Kalman Filtering}

When the system state is constrained to lie on one side of a hyperplane, inequality constrained Kalman filtering [25][28] seeks to update the Kalman state estimate to incorporate this information. One method is to use PDF truncation, which is a technique that will update the filter with the mean and covariance of the region of the PDF that lies outside of the constraint hyperplane [25], [26]. An alternative is to apply a method that we have termed uncertainty projection, which we previously presented in [14]. In this section, we will briefly review our uncertainty projection method for handling inequality constraints.

Let us assume that a Kalman filter, at time-step $k$, is estimating the PDF of a state $\mathbf{x}_{k}$ by a mean vector $\hat{\mathbf{x}}_{k \mid k}$ and covariance matrix $\mathbf{P}_{k \mid k}$. Also, let us assume there is a nonlinear inequality constraint defined as follows,

$$
\phi_{k}^{T} \mathbf{a}\left(\mathbf{x}_{k}\right) \geq \alpha_{k}
$$

where $\alpha_{k}$ is a scalar and $\phi_{k}$ is an $M \times 1$ vector.

Our uncertainty projection method first projects the state estimate to a Gaussian in the constraint space, $\mathbf{s}_{k} \sim$ $\mathcal{N}\left(\hat{\mathbf{s}}_{k}, \mathbf{C}_{k}\right)$, according to $\hat{\mathbf{s}}_{k}=\mathbf{a}\left(\hat{\mathbf{x}}_{k \mid k}\right)$ and $\mathbf{C}_{k}=$ $\mathbf{A}_{k} \mathbf{P}_{k \mid k} \mathbf{A}_{k}^{T}$, where $\mathbf{A}_{k}$ is the Jacobian of $\mathbf{a}\left(\mathbf{x}_{\mathbf{k}}\right)$ evaluated at the current estimate $\hat{\mathbf{x}}_{k \mid k}$. This projection operation makes the constraint linear on the random variable $\mathbf{s}_{k}$,

$$
\phi_{k}^{T} \mathbf{s}_{k} \geq \alpha_{k}
$$

To then update the PDF in the constraint space, we decouple the constraint and apply a one-dimensional PDF update process that is detailed in [14]. Fig. 3 demonstrates this onedimensional constraint update.

Lastly, our algorithm takes the result from the onedimensional update, reverses the transformation that decoupled the constraint, and then projects the new PDF in the constraint space back into the original state space to obtain a new Kalman estimate $\left(\hat{\mathbf{x}}_{k \mid k}^{+}, \mathbf{P}_{k \mid k}^{+}\right)$. An example of our uncertainty projection method applied to a two-dimensional state space is shown in Fig. 2-(b) and more details of the algorithm are in [14].

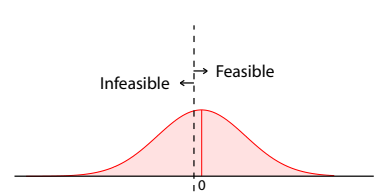

a)

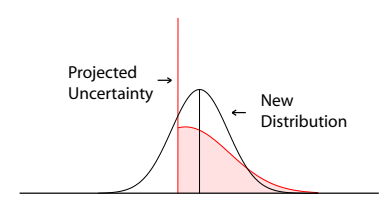

b)
Fig. 3. In (a), a normally distributed random variable is shown with a scalar constraint that defines the feasible and infeasible regions. In (b), we show our uncertainty projection method [14] which projects the tail of the Gaussian onto the constraint and then computes the new mean and variance.

\section{CONSTRAined Filtering FOR SURgicAL ESTIMATION}

Given a preoperative surface model of the surrounding flexible environment and a continuum robot that is exploring the environment with forceful contact, our filtering approach seeks to combine information from on-board position sensing, kinematic models, and geometric constraints to produce accurate registration parameters for localization. This section discusses the overall technical approach including the integration of a contact detection algorithm for multisegment continuum robots [13] and the application of the constrained Kalman filtering algorithms presented in Sec. II to solve this surgical localization problem.

\section{A. Contact Detection}

The robot used for experimental validation is a multibackbone three segment continuum robot [3], [15], see Fig. 1. Each segment is constructed using three circumferentially located super elastic NiTi secondary backbones and one centrally located super elastic NiTi primary backbone. Actuation is achieved by changing the lengths of the secondary backbones in order to actively bend the segments.

The innate compliance of this type of flexible robot allows the robot's natural shape to change due to interaction with an obstacle. By capturing this change in shape, it is possible to detect that a forceful contact with the environment has occurred. To perform this contact detection, we are leveraging algorithms described in [13].

As a consequence of Chasles's theorem [29], the instantaneous motion of the end disk of each segment of the continuum robot is fully described by the Plücker line coordinates of the Instantaneous Screw Axis (ISA) and the screw pitch. The ISA is described by the unit axis $\hat{\boldsymbol{\omega}}_{s}$ and the closest point on the axis to the origin $\mathbf{r}_{s}$ :

$$
\begin{aligned}
\mathbf{r}_{s} & =\frac{\boldsymbol{\omega}_{g_{s} / b_{s}}^{b_{s}} \times\left(\mathbf{v}_{g_{s} / b_{s}}^{b_{s}}+p_{g_{s}}^{b_{s}} \times \boldsymbol{\omega}_{g_{s} / b_{s}}^{b_{s}}\right)}{\left\|\boldsymbol{\omega}_{g_{s} / b_{s}}^{b_{s}}\right\|^{2}} \\
\hat{\boldsymbol{\omega}}_{s} & =\frac{\boldsymbol{\omega}_{g_{s} / b_{s}}^{b_{s}}}{\left\|\boldsymbol{\omega}_{g_{s} / b_{s}}^{b_{s}}\right\|},
\end{aligned}
$$

where $\mathbf{v}_{g_{s} / b_{s}}^{b_{s}}$ and $\omega_{g_{s} / b_{s}}^{b_{s}}$ are the linear and angular velocities of the end disk of segment $s$ with respect to the base disk of segment $s$ respectively. If contact occurs at any intermediate vertebrae between the base of each segment and its base 


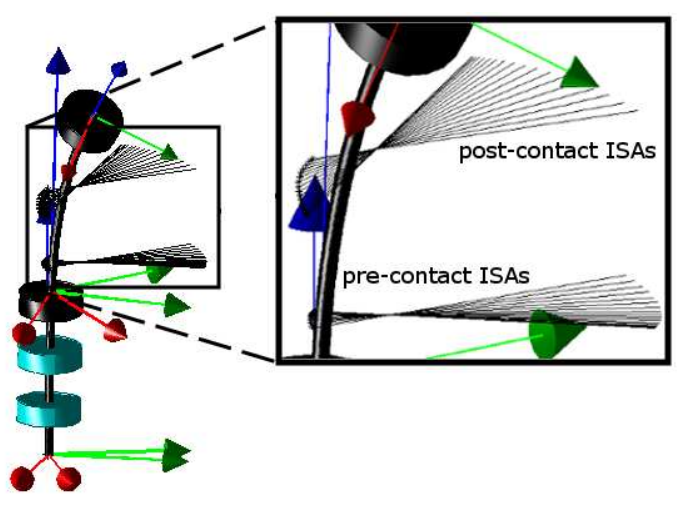

Fig. 4. Group of ISAs after the contact is appreciably shifted toward the end disk of the segment because of the smaller radius of curvature of the unconstrained portion of the segment. The closer the disk in contact is to the end disk, the closer the post-contact ISAs will be to the end disk.

disk, the axode of motion (i.e. the group of infinitesimallyseparated ISA's) shifts toward the end disk of the constrained segment, as shown in Fig 4.

Assume that an extrinsic sensor (such as an electromagnetic tracker) provides the position $\overline{\mathbf{p}}_{g_{s}}^{W}$ and orientation $\overline{\mathbf{R}}_{g_{s}}^{W}$ of the end disk of each segment with respect to a world reference frame $\{W\}$. The relative position, orientation, linear velocity $\overline{\mathbf{v}}_{g_{s} / b_{s}}^{b_{s}}$, and angular velocity $\overline{\boldsymbol{\omega}}_{g_{s} / b_{s}}^{b_{s}}$ of the end disk of segment $s$ with respect to the previous one are given by,

$$
\begin{array}{r}
\overline{\mathbf{p}}_{g_{s}}^{b_{s}}=\overline{\mathbf{R}}_{W}^{g_{s-1}}\left(\overline{\mathbf{p}}_{g_{s}}^{W}-\overline{\mathbf{p}}_{g_{s-1}}^{W}\right) \\
\overline{\mathbf{R}}_{g_{s}}^{g_{s-1}}=\overline{\mathbf{R}}_{g_{s}}^{b_{s}}=\overline{\mathbf{R}}_{W}^{g_{s-1}} \overline{\mathbf{R}}_{g_{s}}^{W} \\
\overline{\mathbf{v}}_{g_{s} / b_{s}}^{b_{s}} \dot{\overline{\mathbf{p}}}_{g_{s}}^{b_{s}} \\
\overline{\boldsymbol{\omega}}_{g_{s} / b_{s}}^{b_{s}}=\operatorname{vect}\left(\overline{\bar{R}}_{g_{s}}^{g_{s-1}} \overline{\mathbf{R}}_{g_{s-1}}^{g_{s}}\right),
\end{array}
$$

where all entities marked with a bar (i.e. $\bar{p}$ ) are based on extrinsic sensory measurements and the operator vect extracts the skew-symmetric vector from a skew-symmetric matrix. By using the theoretical relative position $\mathbf{p}_{g_{s}}^{b_{s}}$, orientation $\mathbf{R}_{g_{s}}^{b_{s}}$, linear and angular velocities, $\mathbf{v}_{g_{s} / b_{s}}^{b_{s}}$ and $\boldsymbol{\omega}_{g_{s} / b_{s}}^{b_{s}}$ of the robot [30], it is possible to define the following Screw Motion Deviation:

$$
\mu_{s}=\left\|\mathbf{r}_{s}-\overline{\mathbf{r}}_{s}\right\|
$$

where $\overline{\mathbf{r}}_{s}$ is calculated using (6) based on sensor kinematics and $\mathbf{r}_{s}$ is calculated using (6) based on the theoretical robot kinematics. Contact is therefore detected independently for any segment when $\mu_{s}>\epsilon_{s}$ for any of the three segments $s=1,2,3$.

\section{B. Filter Formulation}

While an initial guess may exist, the exact parameters that define the registration of the continuum robot to the flexible structure are unknown at the start of an experiment. Using a stochastic representation in the form of a Kalman filter, we seek to estimate these parameters recursively. The Kalman state that our filtering algorithm estimates, at time-step $k$, is comprised of registration parameters and the configuration of the robot, as follows,

$$
\mathbf{x}_{k}=\left[\begin{array}{lllll}
\mathbf{t}^{T}, & \alpha, & \beta, & \gamma, & \boldsymbol{\psi}_{k}^{T}
\end{array}\right]^{T} .
$$

The translation vector $\mathbf{t}$ essentially defines the location of the robot origin in the coordinate frame of the preoperative surface model. Also, $\alpha, \beta$, and $\gamma$ are the roll, pitch, and yaw, respectively, that define the transformation from the robot frame to the surface model. The vector $\boldsymbol{\psi}_{k}$ is the configuration of the robot, as follows,

$$
\boldsymbol{\psi}_{k}=\left[\boldsymbol{\psi}_{1}^{T}, \boldsymbol{\psi}_{2}^{T}, \boldsymbol{\psi}_{3}^{T}\right]^{T},
$$

where each $\boldsymbol{\psi}_{s}$ for $s=1,2,3$ defines the shape of each segment of the continuum robot and is parameterized by two angles: a bending angle $\theta_{L_{s}}$ and an angle that defines the plane in which the bending occurs $\delta_{s}$, thus $\boldsymbol{\psi}_{s}=\left[\theta_{L_{s}}, \delta_{s}\right]^{T}$.

\section{Equality Constraints}

When the screw motion deviation in Eq. 11 exceeds a threshold and the robot is thus declared to be in contact with the flexible environment, this equates to a constraint on where the robot can be (the robot must be touching the surface model). This constraint can be used to eliminate feasible states from the PDF of the Kalman filter. At a first glance, it may seem appropriate to apply a form of soft constraint to the filter estimate when contact is detected due to the possibility of the robot being positioned along a range of states depending on the amount of surface deformation that is expected. If such a constraint could be applied, it would reduce the uncertainty in the filter but will not exactly constrain the robot onto the surface nor will it allow for the inference of the surface deformation.

Instead the solution that we adopt for our experiment is to apply a strict equality constraint to the filter estimate to constrain the filtering problem using our equality constraint update algorithm from Sec. II-A. To do this, we leverage environment stiffness and robot compliance models to express the following equality relationship,

$$
\mathbf{F}_{s}\left(\mathbf{x}_{k}\right)=\mathbf{F}_{r}\left(\mathbf{x}_{k}\right),
$$

where $\mathbf{F}_{s}\left(\mathbf{x}_{k}\right)$ represents the elastic force caused by the deformation of the surface and $\mathbf{F}_{r}\left(\mathbf{x}_{k}\right)$ represents the elastic force caused by the compliance of the robot.

In the context of our equality constraint update algorithm, the appropriate pseudo-measurement function is defined as follows,

$$
g\left(\mathbf{x}_{k}\right)=\left\|\mathbf{F}_{s}\left(\mathbf{x}_{k}\right)-\mathbf{F}_{r}\left(\mathbf{x}_{k}\right)\right\|=0 .
$$

Experimentally, we use a simplified model for $\mathbf{F}_{r}\left(\mathbf{x}_{k}\right)$ that assumes that the force is proportional to the magnitude of the difference between the configuration of the robot, according to the current estimate, and the desired configuration of the robot according to the onboard controller. The parameters for this simplified compliance model of the robot were determined empirically. 


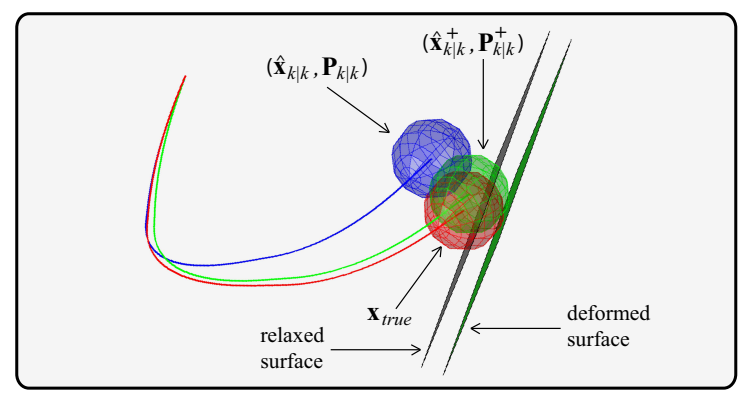

Fig. 5. Equality constrained filtering will move the estimate $\hat{\mathbf{x}}_{k \mid k}$ onto the surface and will properly balance the deformation of the surface with the compliance of the robot. This example shows the new estimate $\hat{\mathbf{x}}_{k \mid k}^{+}$near the ground truth configuration after the constraint update.

In Fig. 5, we show an example of an equality constraint shifting the Kalman estimate $\hat{\mathbf{x}}_{k \mid k}$ so that the new estimate $\hat{\mathbf{x}}_{k \mid k}^{+}$is both contacting and appropriately deforming the surface. In this figure, we have drawn spheres to model the width and shape of the end-effector of the robot. The estimate is shown moving toward the true state $\mathbf{x}_{\text {true }}$ because of the added information provided by the equality constraint.

It is through this equality constrained problem that we can estimate the expected deformation of the surface. Essentially, by inferring the configuration of the robot that properly balances the elastic forces in Eq. 12, we are simultaneously solving for the force that is applied on the surface at the point of contact. Given the stiffness of the material, we can then compute the expected deformation by assuming a linear elastic model. It can be seen in Fig. 5 that determining the configuration of the robot directly provides the surface deformation.

\section{False-Positive Detection}

It is possible for the contact detection algorithm in Sec. IIIA, due to modeling uncertainty and actuator noise, to report false-positives. This means that the equality constraint update procedure might be invoked when in fact the robot is not actually contacting the environment. This could have drastic consequences on the convergence of the filtering algorithm.

To avoid a diverging state estimate, it is important to detect these false-positives, and when one occurs, temporarily ignore the equality constraint that is imposed by the preoperative surface model. To detect false-positives, we can preemptively apply the equality constraint update algorithm assuming the contact detection is correct, and then evaluate the likelihood of the result with a Mahalanobis test,

$$
\left[\hat{\mathbf{x}}_{k \mid k}^{+}-\hat{\mathbf{x}}_{k \mid k}\right]^{T} \mathbf{P}_{k \mid k}^{-1}\left[\hat{\mathbf{x}}_{k \mid k}^{+}-\hat{\mathbf{x}}_{k \mid k}\right]>\zeta .
$$

In Eq. $13, \hat{\mathbf{x}}_{k \mid k}^{+}$is the resulting state mean after performing the equality constraint update, $\hat{\mathbf{x}}_{k \mid k}$ is the prior mean before applying the update, $\mathbf{P}_{k \mid k}$ is the prior covariance, and $\zeta$ is a user chosen threshold. When the Mahalanobis distance exceeds this threshold, we report a false-positive for contact detection. Incorporating this test allows for a more robust estimation process.

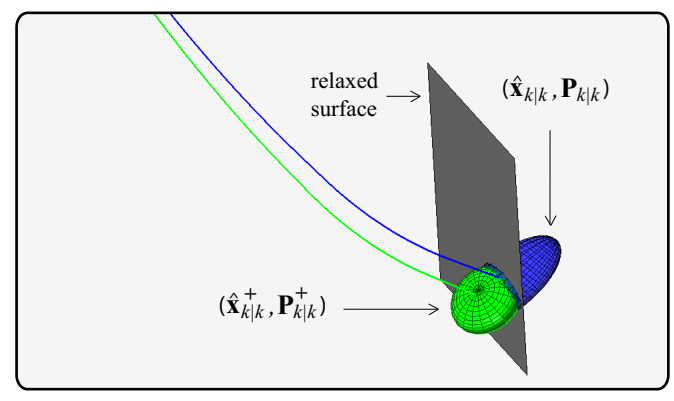

Fig. 6. Inequality constrained filtering can significantly reduce the uncertainty in the state estimate. The prior estimate (blue) is corrected (to green) moving the PDF to the feasible region.

\section{E. Inequality Constraints}

When a false-positive is detected for the contact estimation algorithm based on the likelihood of the filtering estimate, we can assume that the robot is not contacting the environment. Thus, we can say with certainty that the forward kinematic model of the robot must lie outside of the surface model. This equates to an inequality constraint when applied locally.

Assuming that the end-effector position of the robot can be defined as a function of the state, $\mathbf{f}\left(\mathbf{x}_{k}\right)$, according to its kinematic models, we can define local inequality constraints,

$$
\phi_{k}^{T} \mathbf{f}\left(\mathbf{x}_{k}\right)<\alpha_{k},
$$

where $\phi_{k}$ and $\alpha_{k}$ are obtained by assuming the nearest triangular face of the surface model is a plane. The constraint equation forces the end-effector to lie outside of that plane.

In Fig. 6, we show an example of an inequality constraint shifting the estimate so that it lies within the free-space. The ellipses shown in the figure depict the uncertainty of the state (at the end-effector position) before and after the update.

\section{F. Filtering Algorithm}

Alg. 1 is a high-level description of the the entire filtering process. As with a conventional Kalman filter, our algorithm performs a prediction step and a correction step. Then, using the geometric constraints provided by the robot's interaction with the surface model, we perform our novel constraint update procedure to reduce the uncertainty in the Kalman estimate and to resolve the true registration parameters.

\section{EXPERIMENTAL EVALUATION}

The experimental setup is shown in Fig. 1. The robot is a three segment continuum robot with electromagnetic (EM) tracking coils at the base of each segment to measure the local positioning of the robot. There is also an EM tracking coil at the end-effector. The embedded electromagnetic tracking system that we are using is the Ascension Technology 3D Guidance trakSTAR ${ }^{\mathrm{TM}}$ with coils that are $0.9 \mathrm{~mm}$ in diameter. The RMS position error of this system is $1.5 \mathrm{~mm}$ and the RMS orientation error is 0.5 degrees. For this experiment, the end-effector of the robot was driven into forceful contact with a bench-top phantom consisting of a box lined with deformable foam. A Faro 7-axis laser scanner was used to capture an a priori surface model of 

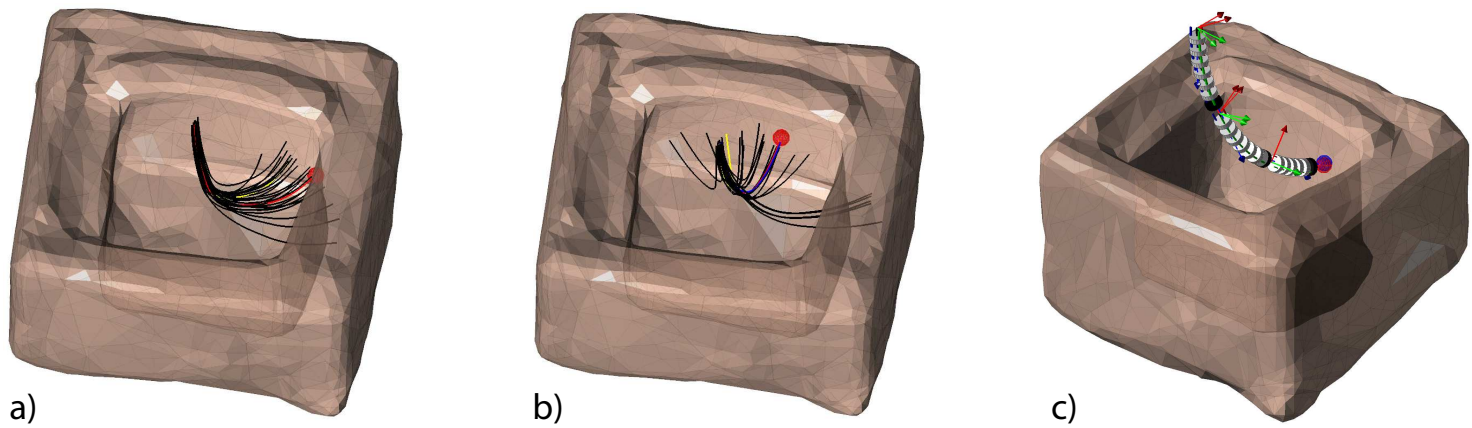

Fig. 7. In (a), multiple hypotheses are shown, each of which runs the constrained filtering algorithm for registration. In (b), some hypotheses have diverged. In (c), the result shows proper alignment between the estimated robot configuration and ground truth.

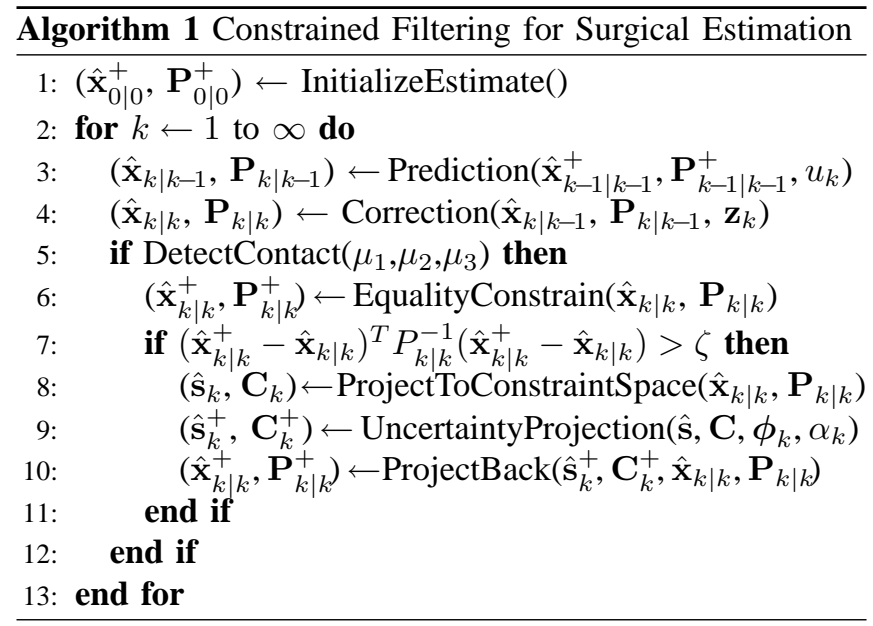

the phantom. The stiffness of the material was measured by assuming axial stiffness of a uniform material with Young's modulus. To obtain ground truth data with which to compare the performance of our localization algorithm, we used a laser scan that captured the relative positioning of the robot to the phantom and extracted the necessary ground truth registration parameters from the geometry of the object.

We ran the continuum robot to random desired target configurations that were out of reach due to the robot being obstructed by the phantom. This forced the robot to contact the surface at many different points. The data was saved and the algorithm was post-processed using Matlab, although we chose to only update the filter when the robot was relatively stable (its velocity was below a threshold). This was to aid in the stability of the filtering algorithm and we note that this is something that we will relax in our future work.

In Fig. 7, we show the result of a registration experiment. In Fig. 7-(a), a set of initialized hypotheses are shown before the data is processed. Each of these hypotheses was evaluated and updated using the constrained filtering algorithms presented in this paper. In Fig. 7-(b), it can be seen that many of the hypotheses for the registration and localization have begun to move toward the ground truth configuration. Some hypotheses, though, have diverged due to a poor initial alignment and the local affect of the filtering updates. In Fig. 7-(c), the resulting configuration of the robot, estimated with our filtering algorithm, is shown overlapping with the ground truth configuration. This estimate emerged with the highest likelihood after imposing all of the constraints due to contact with the environment.

The initial guess for the registration assumed a $14.6 \mathrm{~mm}$ error at the position of the robot end-effector. This equates to an approximately 10 percent error with respect to the length of the robot. The error was mostly associated with a largely erroneous yaw angle. After processing the contact data and applying the constraints with the Kalman filter, our final registration error was reduced to $1.1 \mathrm{~mm}$ (with respect to the end-effector position), which equates to a less than 0.8 percent positional error with respect to the length of the robot. We believe this result demonstrates the feasibility of our approach for accurate registration in compliant environments.

In the experiment, we show that some of the initialized hypotheses diverge over time. While this shows that the individual result of each hypothesis is sensitive to its initialized estimate, it does not suggest that the final performance of the entire filtering scheme is necessarily sensitive to the initial guess for registration. Instead, the divergence of these hypotheses shows that the act of filling the state space with initial hypotheses is in fact serving its purpose: the hypotheses that are somewhat close to the true alignment will converge to the true state and the ones that are out of alignment will drop in likelihood. The significance is that as long as we adequately cover the initial state space with initial hypotheses, the true state will emerge.

\section{CONCLUSIONS}

The goal of the algorithm that we have presented is to aid in the creation of fully representative rendered models for surgical image-guidance. For medical procedures that cannot utilize direct vision, a surgeon can refer to the rendered visualization for visual feedback. Thus, it is important that the surgeon trusts the registration and alignment of the robot relative to the organ surface models.

To solve registration, we present a novel filtering algorithm that leverages geometric constraints within a Kalman filter framework to automatically register and localize a compliant surgical robot with respect to an a priori $3 \mathrm{D}$ deformable surface model. Our method relies on an equality constrained 
update step for when the robot is forcefully contacting the environment and an inequality constrained update step for when a false-positive is detected and thus the robot is lying in the free-space of the environment. With this filtering procedure, we can incrementally eliminate regions of the state space that are decidedly infeasible, eventually resolving the true state that defines the registration of the robot. The theoretical contributions of this work are its use of equality constrained Kalman filtering to localize a surgical robot and the consideration of elastic force models within a Kalman filter for applying equality constraints.

For future work, we will incorporate an improved compliance model for the robot that relaxes our somewhat over-simplified model for computing $\mathbf{F}_{r}\left(\mathbf{x}_{k}\right)$ in Eq. 12. Despite our simplified model, though, the filtering algorithm performed well and localized the robot. We attribute the success to the inherent stochasticity of the approach, which means that despite noisy sensing and imperfect models, there was still enough information acquired through constraints to produce a likely final registration. Also for future work, we will adopt a more sophisticated stiffness model for the environment. For this paper, we assumed a linear model but admit that when modeling tissue for surgical applications, a linear stress-strain relationship is less than ideal.

We have shown with a successful experiment that accurate registration can be achieved automatically using our proposed algorithm. In the future, we intend to test this algorithm with in vivo experiments and will also extend the approach to account for uncertainty in the a priori surface models. Future experiments will focus on testing the robustness of our approach under real-time conditions, realistic environments, varying stiffnesses, and organ movement.

\section{REFERENCES}

[1] A. Degani, H. Choset, A. Wolf, and M. Zenati, "Highly articulated robotic probe for minimally invasive surgery," in Robotics and $\mathrm{Au}$ tomation, 2006. ICRA 2006. Proceedings 2006 IEEE International Conference on, May 2006, pp. 4167-4172.

[2] A. Degani, H. Choset, A. Wolf, T. Ota, and M. Zenati, "Percutaneous intrapericardial interventions using a highly articulated robotic probe," in Biomedical Robotics and Biomechatronics, 2006. BioRob 2006. The First IEEE/RAS-EMBS International Conference on, Feb. 2006, pp. $7-$ 12.

[3] A. Bajo, R. Goldman, and N. Simaan, "Configuration and joint feedback for enhanced performance of multi-segment continuum robots," in Robotics and Automation (ICRA), 2011 IEEE International Conference on, May 2011, pp. 2905-2912.

[4] L. Joskowicz, "Fluoroscopy-based navigation in computer-aided orthopaedic surgery," in Proc. of the IFAC Conf. on Mechatronic Systems., 2000.

[5] R. Omary, J. Green, B. Schirf, Y. Li, J. Finn, and D. Li, "Realtime magnetic resonance imaging-guided coronary catheterization in swine," Circulation, vol. 107, no. 21, pp. 2656-2659, 2003.

[6] R. Manzke, V. Reddy, S. Dalal, A. Hanekamp, V. Rasche, and R. Chan, "Intra-operative volume imaging of the left atrium and pulmonary veins with rotational x-ray angiography," in Medical Image Computing and Computer-Assisted Intervention (MICCAI), 2006, pp. 604-611.

[7] W. Zhang, R. Rohling, and D. K. Pai, "Surface extraction with a threedimensional freehand ultrasound system," Ultrasound in Medicine and Biology, vol. 30, no. 11, pp. 1461-1473, 2004.

[8] K. Cleary, H. Zhang, N. Glossop, E. Levy, B. Wood, and F. Banovac, "Electromagnetic tracking for image-guided abdominal procedures: Overall system and technical issues," in Engineering in Medicine and Biology Society, 2005. IEEE-EMBS 2005. 27th Annual International Conference of the, 2005, pp. 6748-6753.
[9] W. Wein, A. Khamene, D. Clevert, O. Kutter, and N. Navab, "Simulation and fully automatic multimodal registration of medical ultrasound," in Medical Image Computing and Computer-Assisted Intervention (MICCAI), 2007, pp. 136-143.

[10] H. Zhong, T. Kanade, and D. Schwartzman, "Sensor guided ablation procedure of left atrial endocardium," in Medical Image Computing and Computer-Assisted Intervention (MICCAI), 2005, pp. 1-8.

[11] J. Dong, H. Calkins, S. Solomon, S. Lai, D. Dalal, A. Lardo, E. Brem, A. Preiss, R. Berger, H. Halperin, and T. Dickfeld, "Integrated electroanatomic mapping with three-dimensional computed tomographic images for real-time guided ablations," Circulation, vol. 113, pp. 186194, 2006.

[12] A. B. Koolwal, F. Barbagli, C. Carlson, and D. Liang, "An ultrasoundbased localization algorithm for catheter ablation guidance in the left atrium," The International Journal of Robotics Research, vol. 29, no. 6, pp. 643-665, May 2010.

[13] A. Bajo and N. Simaan, "Kinematics-based detection and localization of contacts along continuum robots," IEEE Transaction on Robotics, vol. 99, no. 2, 2012.

[14] S. Tully, G. Kantor, and H. Choset, "Inequality constrained Kalman filtering for the localization and registration of a surgical robot," in Intelligent Robots and Systems (IROS), 2011 IEEE/RSJ International Conference on, Sept. 2011, pp. 5147-5152.

[15] A. Bajo and N. Simaan, "Finding lost wrenches: Using continuum robots for contact detection and estimation of contact location," in Robotics and Automation (ICRA), 2010 IEEE International Conference on, May 2010, pp. 3666-3673.

[16] D. Simon, "Kalman filtering with state constraints: a survey of linear and nonlinear algorithms," Control Theory Applications, IET, vol. 4, no. 8, pp. 1303-1318, August 2010.

[17] M. Tahk and J. Speyer, "Target tracking problems subject to kinematic constraints," IEEE Transactions on Automatic Control, vol. 35, no. 3, pp. 324-326, March 1990.

[18] A. Alouani and W. Blair, "Use of a kinematic constraint in tracking constant speed, maneuvering targets," IEEE Transactions on Automatic Control, vol. 38, no. 7, pp. 1107-1111, July 1993.

[19] L. Wang, Y. Chiang, and F. Chang, "Filtering method for nonlinear systems with constraints," Control Theory and Applications, IEEE Proceedings, vol. 149, no. 6, pp. 525-531, November 2002.

[20] B. Teixeira, J. Chandrasekar, L. Torres, L. Aguirre, and D. Bernstein, "State estimation for equality-constrained linear systems," in Decision and Control, IEEE Conference on, December 2007, pp. 6220-6225.

[21] N. Gupta, "Kalman filtering in the presence of state space equality constraints," in Chinese Control Conf., December 2007, pp. 107-113.

[22] D. Simon and T. Chia, "Kalman filtering with state equality constraints," IEEE Transactions Aerosp. Electron. Systems, vol. 39, no. 1, pp. 128-136, January 2002.

[23] S. Julier and J. LaViola, "On kalman filtering with nonlinear equality constraints," Signal Processing, IEEE Transactions on, vol. 55, no. 6, pp. 2774-2784, June 2007.

[24] B. Bell and F. Cathey, "The iterated kalman filter update as a gaussnewton method," IEEE Transactions on Automatic Control, vol. 38, no. 2, pp. 294-297, 1993.

[25] D. Simon and D. L. Simon, "Constrained kalman filtering via density function truncation for turbofan engine health estimation," Int. Journal of Systems Science, vol. 41, no. 2, pp. 159-171, February 2010.

[26] N. Shimada, Y. Shirai, Y. Kuno, and J. Miura, "Hand gesture estimation and model refinement using monocular camera - ambiguity limitation by inequality constraints," in Proc. The 3rd Int. Conference on Automatic Face and Gesture Recognition, 1998, pp. 268-273.

[27] B. O. Teixeira, L. A. Trres, L. A. Aguirre, and D. S. Bernstein, "On unscented kalman filtering with state interval constraints," Journal of Proc. Control, vol. 20, no. 1, pp. 45-57, 2010.

[28] P. Vachhani, S. Narasimhan, and R. Rengaswamy, "Robust and reliable estimation via unscented recursive nonlinear dynamic data reconciliation," Journal of Proc. Control, vol. 16, no. 10, pp. 1075-1086, 2006.

[29] J. Angeles, "Automatic computation of the screw parameters of rigidbody motions. part ii: Infinitesimally- separated positions," Journal of Dynamic Systems, Measurement, and Control, vol. 108, no. March, pp. 39-43, 1986.

[30] N. Simaan, R. Taylor, and P. Flint, "A dexterous system for laryngeal surgery," in Robotics and Automation (ICRA), 2004 IEEE International Conference on, New Orleans, LA, 2004, pp. 351-357. 